\title{
A Low Cost Testbed and Test-Design Methodology for Nanosatellite Sub-/Systems
}

\author{
Yaseen Zaidi \\ French South African Institute of Technology \\ Dept. of EE\&CE \\ Cape Peninsula University of Technology \\ Bellville, South Africa 7530 \\ yaseen.zaidi@ieee.org
}

\author{
Robert van Zyl \\ French South African Institute of Technology \\ Dept. of EE\&CE \\ Cape Peninsula University of Technology \\ Bellville, South Africa 7530 \\ vanzylr@cput.ac.za
}

\begin{abstract}
The architecture of a software-driven testbed is discussed. The test setup has been developed as measurement and instrumentation system to functionally verify and thermally validate the communication subsystems designed and manufactured for the nanosatellite applications. The system is applied for qualification of a communications subsystem currently under consideration for ZA-CUBE-2 mission.
\end{abstract}

Keywords-Automation, Communication, Linearity, Measurement, Nanosatellite, Testbed.

\section{INTRODUCTION}

The cube and nanosatellite phenomenon is a disruptive technology that continues to lead to new technological developments. The technology is also a change agent in the traditional, elite space industry which in past had lend itself to expensive missions of high commercial or scientific value, built with highly reliable, usually space-grade parts. The cube and nanosatellites are built with the commercial off the shelf grade components, usually of industrial temperature range. The failure rate of these satellites is extremely high [1], especially of the academic missions. Test campaign of the cube or nano missions is a critical aspect of the project to ensure their survival and successful operation in the space environment.

We report the engineering effort undertaken at the French South African Institute of Technology (F'SATI) to realize a methodology for functional verification and limited environmental validation, as well as a setup to qualify various subsystems and payloads currently in development for the ZACUBE-2 mission (Fig. 1), a 3U cubesat.

\section{Background And Space Mission Assurance}

ZA-CUBE-2 is a precursor mission to Operation Phakisa [2], an initiative of the government of South Africa aiming to ocean monitoring and e-governance through low cost nanosatellites assisting in the Maritime Domain Awareness at the tip of the African continent. The mission's primary objective is to evaluate and demonstrate automatic identification system based RF communication and optical payload technologies for earth observation before a fully-fledged maritime nanosatellite constellation is launched.

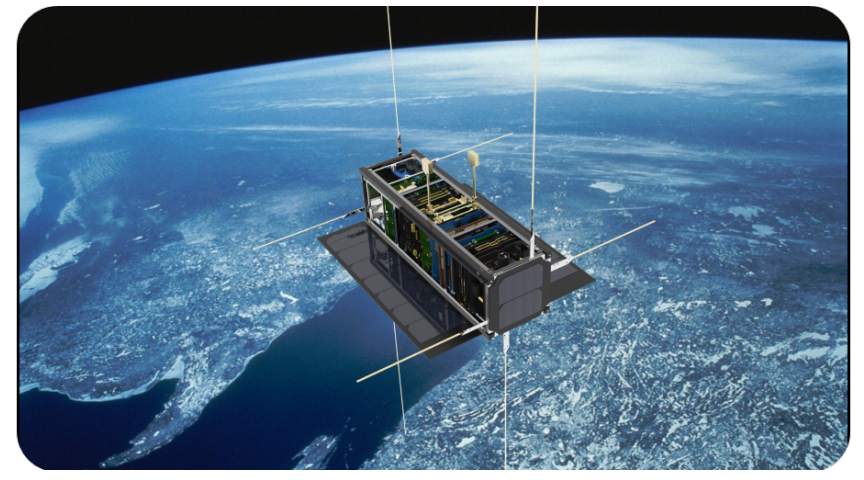

Fig. 1: ZA-CUBE-2 mission concept, the nanosatellite will carry a communications and two optical payloads.

The nanosatellite mission development is characterized by decisions in mission objectives, system requirements, payload/bus configurations and launcher selection that are often revised late in the engineering cycle. In order to accelerate the development and to readily adjust to the project dynamics, F'SATI, through a systems engineering approach, is proceeding to develop a mission assurance facility. The facility is envisioned for cutting the engineering cycle by overlapping the iterative phases of design, development, verification and environmental validation, as soon as the prototypes are available. Functional testing of assembled and integrated hardware and software units can be performed rapidly, and thereafter subjected to environmental qualification. The facility is conceived to achieve high coverage of design verification.

From the success and the lessons learned through the ZACUBE-1 mission, the expertise and legacy equipment are being reused to piece together a test automation setup in a phased way. This approach is adopted to avoid a hefty and immediate one-time investment. In the meantime, technical needs analysis is underway for the procurement of the remaining elements that will upgrade the facility to greater compliance toward space level qualification. The priority of future qualification is on mechanical loads and thermal-vacuum cycling loads.

Since a typical nanosatellite project is a short one compared to the commercial, high-end space missions, co-engineering is the preferred applied systems engineering approach in which 


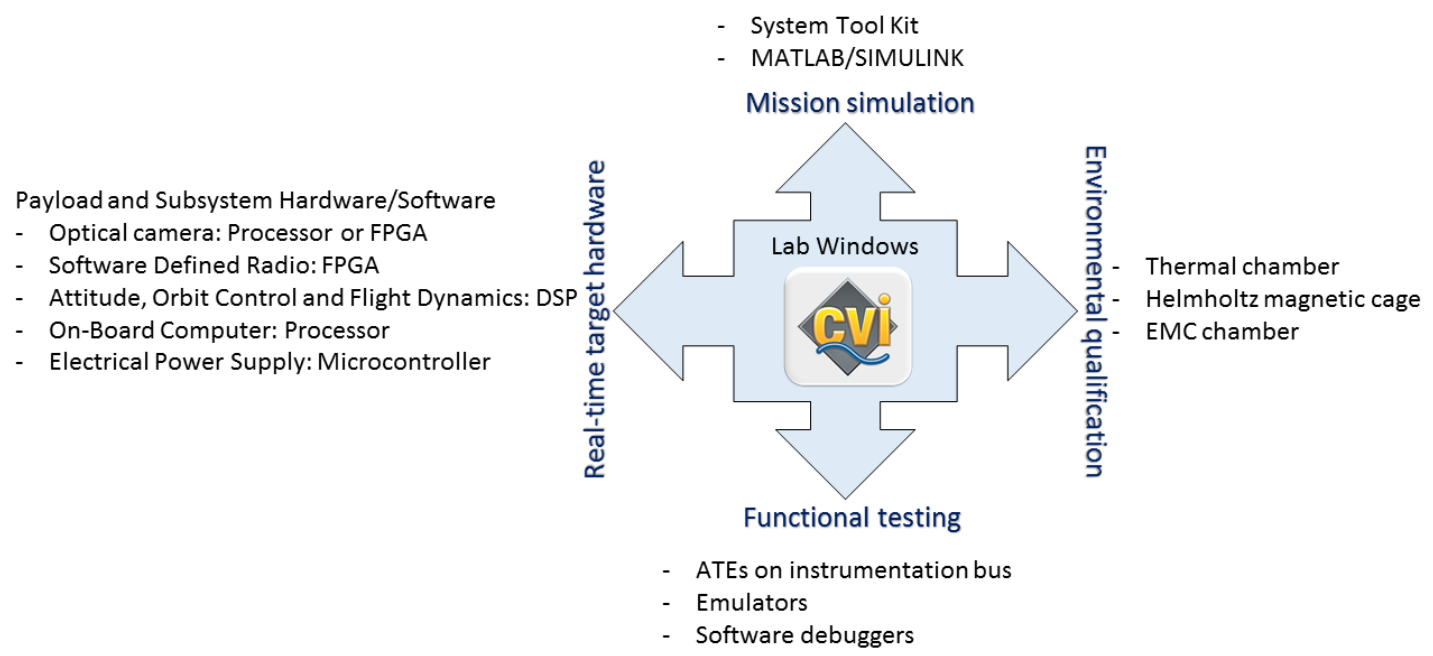

Fig. 2: 'C' language heterogeneous interfaces support integrated design and test through simulator coupling, real-time target, functional verification and climate validation.

mission concept, requirements engineering, system/subsystem and physical design, testing, and verification and validation can be orchestrated either concurrently or with overlap, and hence rework and iteration can be reduced.

The methodology shown in Fig. 2 allows a common development platform for the unification and coordination of the aforementioned activities. Owing to the $\mathrm{C}$ nativity, the topology allows a multitude of engineering phases in the nanosatellite development i.e., system simulation, embedded design, functional verification and environmental validation. For example, the connectivity to the system controller computer running NI LabWindows/CVI API can be physically distributed i.e., by way of TCP sockets to couple simulation data. The much valuable data from live simulation of mission control algorithms, attitude and orbit perturbations, magnetic fields as observed by the satellite or thermal load profile during orbit propagation can be imported to the test and development scenarios. Similarly, flight hardware using embedded processors can be developed and integrated to the test and measurement system along with the mission simulation.

The focus of the present work is on verification and validation depicted by the right and bottom arrows in Fig. 2 .

\section{RELATED WORK}

Automation is usually applied in spacecraft development to a specific subsystem or module. Many groups have applied automation toward algorithm development with hardware/software-in-the-loop testbeds such as [3] and [4]. Our approach provides support for modeling, development, testing and functional and thermal qualification on a single platform and eases the tedious processes related to environmental test and qualification [5][6]. The work shown in [7] uses Python at application layer level, while we address a broader scope with the development around $\mathrm{C}$, starting from instrumentation and measurements [8]. With the setup we

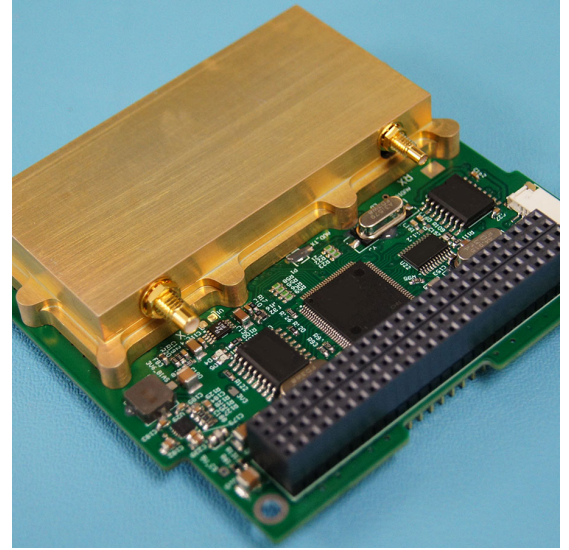

Fig. 3: VHF and UHF transceiver as communication subsystem in standard $10 \times 10 \mathrm{~cm}$ cubesat form factor.

study several classes of communication performance figures of merit [9][10][11][12].

\section{Testbed Architecture}

The hardware and software architecture of the testbed has been developed to assist, at the moment, in the Phase-D of space project. The testbed performs physical layer level functional tests of the RF communication payloads or subsystems. Fig. 3 is an example of a Device Under Test (DUT), a telemetry and telecommand transceiver (CMC) which was flown on ZA-CUBE-1. The DUT may be connected to a variety of test and measurement equipment as illustrated in Fig. 4.

An important element of testbed is a testware, called Missurance, a Graphical User Interface (GUI) suite developed in CVI. This application is designed due to ease of software development support and the availability of huge cache of instrument drivers. Furthermore, the C-interface allows inte- 


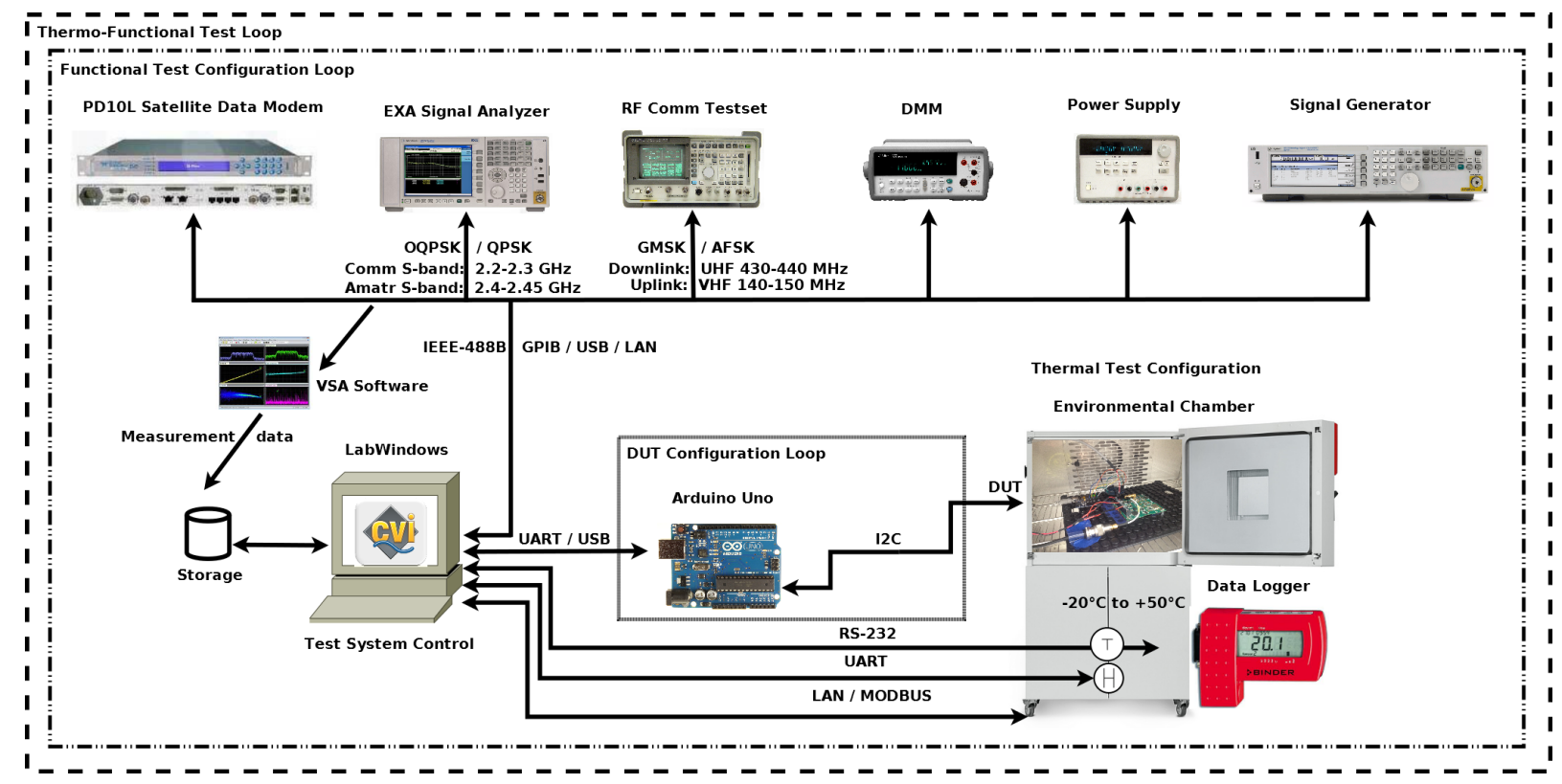

Fig. 4: Testbed for nanosatellite payload and subsystem qualification, shown with communication interfaces and protocols to the DUT, ATEs and the environmental chamber.

gration of simulation, development, verification and validation capabilities.

\section{A. Instrumentation Bus}

Due to the variety of interfaces in the Automated Test Equipment (ATE), the bus supports high accessibility of the measurements: IEEE-488.2/General Purpose Interface Bus (GPIB), USB, LAN, UART and RS-232. The thermal chamber communicates on Modbus protocol over TCP/IP. Humidity is measured via over the air wireless interface. A dashboard based on Arduino sets up the baseband block the DUT via $\mathrm{I}^{2} \mathrm{C}$ interface as shown in Fig. 4. Such re-configuration of the DUT is required during the thermal test to verify all modes of the DUT operation over the temperature cycle.

\section{B. Event Driven Programming}

Missurance is developed in event driven programming. Events are graphical controls on the GUI. Since the goal of test automation is full autonomy, all events are deterministic and user generated. When the events occur, i.e., un/selected, they are properly handled by registering their callbacks. All graphical controls and functions are organized in the calls shown to the left in Fig. 5.

It has been a goal also to provide the user with the minimal decision making capability by limiting the number of graphical controls on the GUI in configuring a test. Therefore, most of the navigation or configurability whether of the instrument or the test tone has been kept hidden, and encapsulated in the underlying callback function of the particular control on the GUI. This can be seen on the right side of Fig. 5 which is a tab for a advanced signal analyzer that has its own CPU running Vector Signal Analysis (VSA) software.

\section{Abstraction Levels}

The access to the testsets consists of a mix of abstraction levels: C driver, Virtual Instrument Software Architecture (VISA) and Standard Commands for Programmable Instruments (SCPI). The VISA software driver architecture aims to codify software standard for major physical layers and protocols of the test and measurement industry whether RS232/422/485, GPIB and GPIB encapsulated interfaces e.g., Ethernet/TCP/IP, serial or USB, and high speed I/O interfaces such as VXI and PXI, typically used in production or manufacturing level tests for acquisition of large number of channels. The VISA commands are lower-level feature access of the testsets and require grouping of several commands to perform complex tasks required in a specific test. The SCPI framework on the other hand is a program level movement by the SCPI Consortium to standardize syntax to construct complex string structures to commanding the test equipment. This level of abstraction boils down to the simplest and most primitive terms of using ASCII style commands. A C-level driver is a higher level instrument access mechanism. In effect, the driver encapsulates the SCPI commands automatically before sending them. However, a $\mathrm{C}$ driver typically implements the most widely used tests and thus cannot perform every test function possible of the ATE. To circumvent this limitation, the crude SCPI command set may be used for fine control of the instruments. Generally, the test equipment always respond to the SCPI level calls even if the other abstraction levels fail to render response.

The diversity in abstraction is a necessity to guarantee compatibility with the ATE standards and interoperability of the ATE to the system controller computer. The command layer for any abstraction level is above IEEE-488.2 communication 

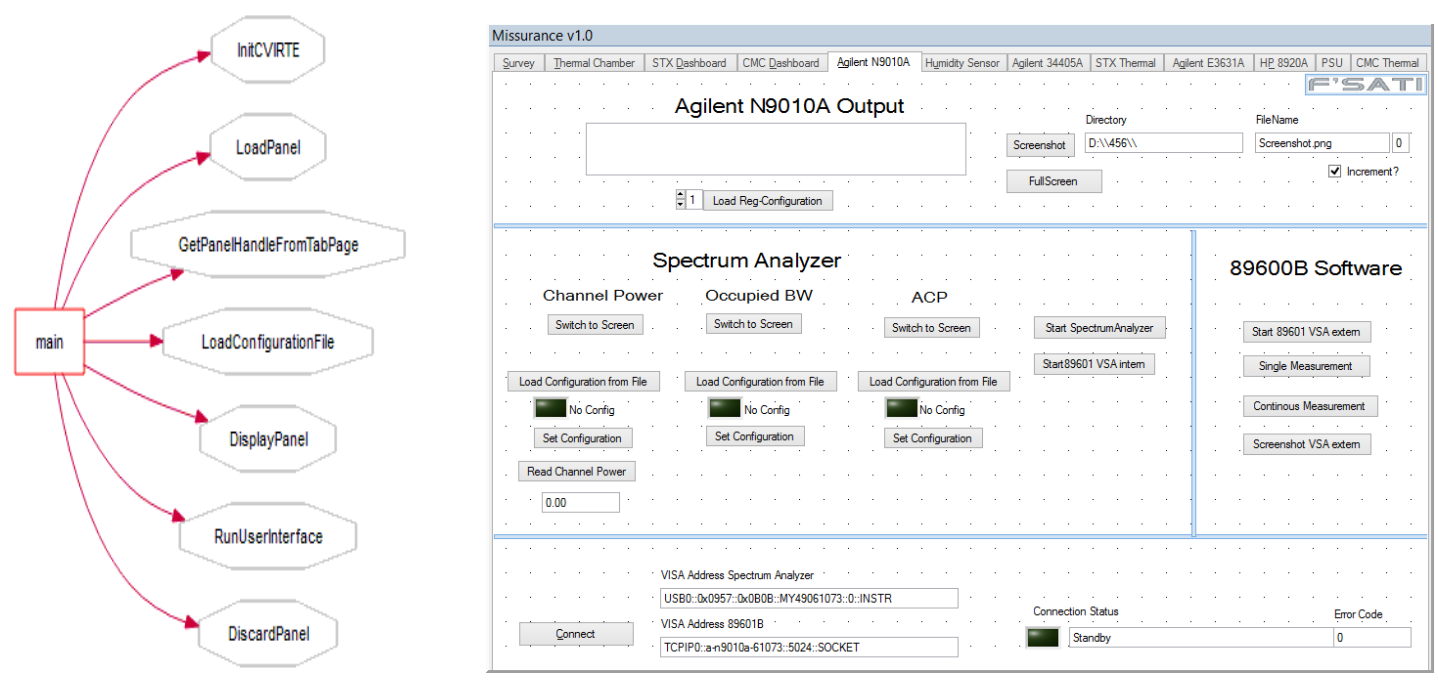

Fig. 5: UI calls to functions and a tab for tests related to the signal analyzer.

Listing 1 Abstraction levels for different instrument control and measurements

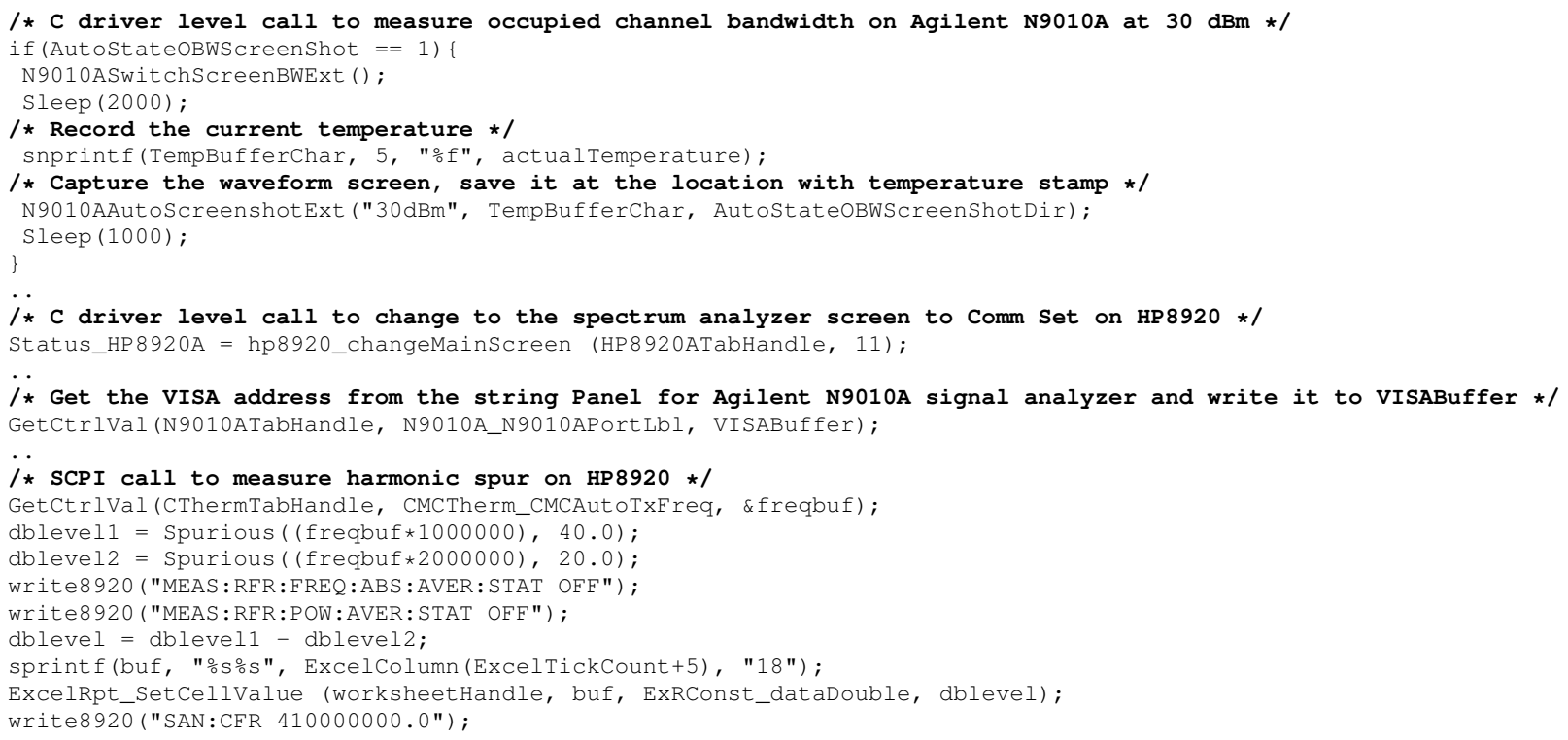

layer, which is above the transmission standard variety of the physical layer [13]. Listing 1 formulates the general concept of the abstraction levels.

\section{Test Control Logic}

The routine in Algorithm 1 is used for DUT configuration and re-configuration. Algorithm 2 refers to the test control logic applied to three loops shown in Fig. 4 in the dotted blocks. Essentially, the DUTs is configured in its default mode first in the inner block and the concerned testsets are initialized and test signals are programmed. Then, in the middle block, the functional tests are performed in each mode of the DUT operation. The outer loop controls the thermal cycle in which the functional behavior is assessed. At the end of each thermal cycle, the DUT mode is changed to repeat the functional and thermal tests. The measurements on legacy ATEs such as in HP8920 UHF/VHF testset and other generic instruments are recorded in tabular format. Whereas, on the advanced testsets, the entire signal spectrum is acquired as image at the specific temperature.

One of the difficulties in precisely determining the in-orbit variation of the thermal loads is the uncertainty in orbital heating. This is largely attributed to the highly unpredictable solar weather. In the nanosatellite arena, the testing at acceptablelevel within the commercial temperature range is generally preferred. Our thermal test specification consists of a single thermal cycle range over $-20^{\circ} \mathrm{C}$ to $+50{ }^{\circ} \mathrm{C}$. Each step is a $+/-10^{\circ} \mathrm{C}$ and at which the thermal bake out lasts $10 \mathrm{~min}$. 

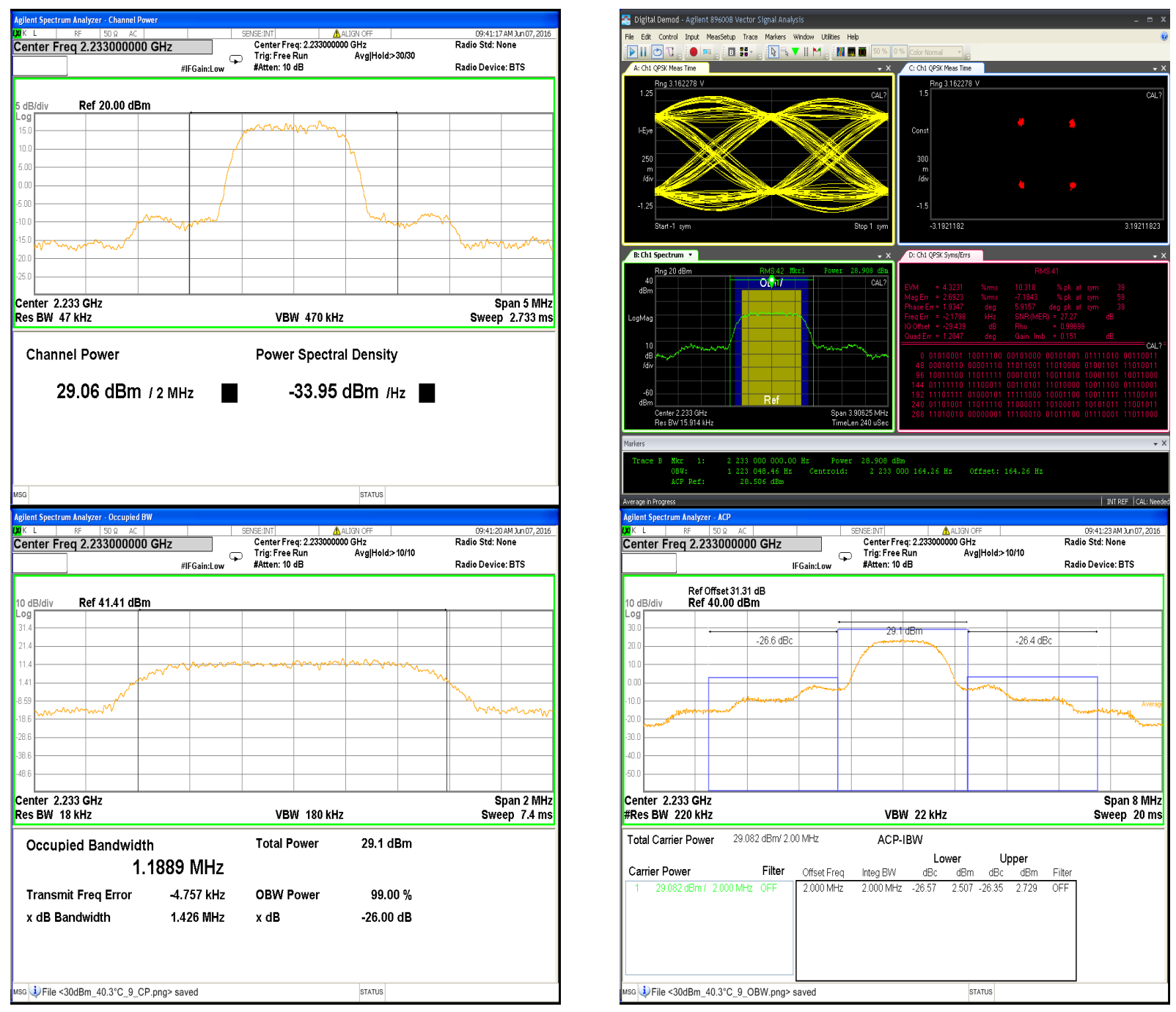

Fig. 6: Vector signal measurements are recorded at $40.3^{\circ} \mathrm{C}$. From top left: channel's RF power, VSA measurements, adjacent channel power (bottom right) and occupied channel bandwidth.

\section{Vi. Measurement Test Results}

The testbed is applied to verify and validate a radio operating in $2.2 \mathrm{GHz}$ to $2.4 \mathrm{GHz}$ range at $24 \mathrm{dBm}$ to $30 \mathrm{dBm}$ transmit power levels. The modulation can be programmed as QPSK or OQPSK in the digital radio. This system, called STX, conforms to CubeSat Standard; it can be used for $1 \mathrm{Mbps}$ downlink for imagery or other high-speed scientific payload data.

Fig. 6 illustrates the vector signal measurement waveforms and numerical data. The top left figure shows the corresponding transmitting power of the channel. The top right plot gives an array of VSA measurements such as the error vector magnitude, errors in frequency, phase, gain, IQ offset and the quadrature error. All these metrics reveal some degree of channel's linear response, QPSK sensitivity to noise and efficiency of the modulation. In the STX system, linearity of QPSK is imperative which is measured by the IQ signal immunity to nonlinear noise as the spectrum tends to regenerate outside the passband. The bottom left and right plots are the Occupied
Channel Bandwidth (OCBW) and Adjacent Channel Power (ACP) measurements respectively showing the content of out of band spectral density.

TABLE I: STATISTICAL SUMMARY OF RF CHANNEL CURRENT

\begin{tabular}{|c|l|l|l|l|}
\cline { 2 - 5 } \multicolumn{1}{c|}{} & \multicolumn{4}{c|}{ Channel Current (A) } \\
\hline RF Power $(\mathrm{dBm})$ & Max & Min & Mean & Std. Dev. \\
\hline 24 & 0.4707 & 0.4684 & 0.4698 & 0.0007 \\
26 & 0.5371 & 0.5278 & 0.5322 & 0.0032 \\
28 & 0.6442 & 0.6237 & 0.6345 & 0.0069 \\
30 & 0.7998 & 0.7275 & 0.7823 & 0.0240 \\
\hline
\end{tabular}

Table I shows a slight digression in the transmitter's DC current over the thermal cycle. At $30 \mathrm{dBm}$ the current swing in cycle indicates that the RF Power Amplifier (PA) is driven hard at this level, close to saturation and just below the nonlinear region, and therefore, the bias point must also shift to the high side to maximize the output power. This is the point where amplifier efficiency is the highest and the linearity is pushing 
the limit. The standard deviation in DC current indicates that from stability of bias standpoint the best operation mode is $24 \mathrm{dBm}$ at which the deviation is the least and thus the RF performance is steady-state and amplifier is truly linear over the thermal cycle. However, the $24 \mathrm{dBm}$ power level may be deemed inadequate in fading channel conditions, at higher orbital altitudes or under poor antenna coverage. On the contrary, from conversion point of view, the desirable range is $28 \mathrm{dBm}-30 \mathrm{dBm}$, provided the thermal design margin in the spacecraft can accommodate for the rise in current, the appearance of nonlinearities and other spurious character of the transmission due to the deviation in DC bias drive.

TABLE II: STATISTICAL SUMMARY OF RF CHANNEL POWER

\begin{tabular}{|l|l|l|l|l|}
\hline RF Power $(\mathrm{dBm})$ & Max & Min & Mean & Std. Dev. \\
\hline \hline 24 & 25.16 & 24.67 & 24.89 & 0.1291 \\
26 & 26.75 & 26.37 & 26.52 & 0.1217 \\
28 & 28.71 & 28.36 & 28.54 & 0.0895 \\
30 & 30.71 & 29.86 & 30.39 & 0.2766 \\
\hline
\end{tabular}

Table II gives statistical summary of deviation in the transmit power sweep over the thermal cycle and confirms strong stability in the output power. From the RF power standpoint, the most stable mode over the thermal cycle is at $28 \mathrm{dBm}$. At low temperatures the output power levels still hold, therefore, the transmitter may be operated via battery in eclipse cycle or during the night passes. At high temperatures, there tends to be a decline in output power, which is more prominent at higher power levels. This is a mix effect due to PA operation at the edge of linearity limit and wavering of the bias due to self-heating of the amplifier as well as the high ambient temperature.

TABLE III: STATISTICAL SUMMARY OF THE RF WAVEFORM PROPERTIES

\begin{tabular}{|l|l|l|}
\hline 24 dBm Input & Mean & Std. Dev. \\
\hline \hline Error Vector Magnitude (EVM) (\% rms) & 5.7223 & 0.1243 \\
Mag Error (\% rms) & 3.7223 & 0.2476 \\
Phase Error (deg) & 2.4716 & 0.0749 \\
Freq. Error (kHz) & -2.1677 & 0.6796 \\
IQ Offset (dB) & -31.4463 & 0.4900 \\
Quad Error $(m$ deg) & 747.0716 & 107.3915 \\
IQ Gain Imbalance $(\mathrm{dB})$ & 0.0909 & 0.0101 \\
Wave Quality $(\rho)$ & 0.9960 & 0.0001 \\
SNR / MER $(\mathrm{dB})$ & 24.8439 & 0.1875 \\
\hline
\end{tabular}

Table III gives statistical interpretation of the thermal stability in key performance metrics. The waveform data is shown in top right in Fig. 6. The linear QPSK modulation is highly sensitive to phase noise typically appearing in the PA. The phase error is minor which shows linear behavior of the RF channel (mixer and the PA). The QPSK modulation doubles the data rate and halves the occupied bandwidth by splitting the data stream in I and $\mathrm{Q}$ paths. However, the doing introduces errors as the offset, in the IQ gains and the quadrature multiplier and, thus, the whole modulation scheme is affected due to circuit imperfections and sensitivity of the signal paths over temperature. In an ideal channel the balance given by Eq. 1 would be $0 \mathrm{~dB}$.

$$
I Q \text { Gain Imbalance }=20 \log _{10}\left[\frac{I}{Q}\right]
$$

The errors in I and Q are also aggregately measured as Error Vector Magnitude (EVM). Note the low standard deviation in I, Q related measurements in Table III. The metrics reveal the distortion in transmitted tone from the ideal test tone which may be quantified by another metric, the waveform quality factor $(\rho) . \rho$ is a correlation factor of the measured tone with the ideal tone energy.

$$
\rho=\frac{\left|\sum_{n} x_{1}(n) x_{2}(n)^{*}\right|^{2}}{\sum_{n}\left|x_{1}(n)\right|^{2} \sum_{n}\left|x_{2}(n)\right|^{2}}
$$

where $x_{2}(n)^{*}$ is the complex conjugate of the measured constellation vector $x_{2}(n)$ and $x_{1}(n)$ is the complex envelope of the ideal constellation. In land mobile systems such as CDMA (IS-95) the specification requirement for $\rho$ is generally $95 \%$ or above for superior transmission quality. $\rho$ is related to EVM ans SNR as

$$
\begin{aligned}
& \rho=\frac{1}{E V M^{2}+1} \\
& S N R=\frac{\rho}{1-\rho}
\end{aligned}
$$

Table IV gives the statistical summary of ACP and OCBW for transmission at all power levels over one thermal cycle. The stability of carrier frequency is given by the standard deviation between the high and cold temperature extremes and high and low RF power levels.

In the subscriber based transmissions e.g., LTE and WCDMA, ACP is a stringent regulatory requirement for ensuring service quality to the neighboring users, although channels may be geographically separated. In the nanosatellites applications, ACP and OCBW are restricted to meet the frequency and power requirements imposed by the frequency management authority responsible for spectrum sharing, and also for minimizing RFI and susceptibility to the other subsystems of the spacecraft itself. As such, the ACP emissions could have grave effects on any high-speed digital module e.g., on-board computer and back-end electronics of the imager, electric power circuits and magnetic device such as the magnetometer and magnetorquer rods required for spacecraft attitude determination and correction.

\section{CONCLUSION}

The testbed development activity is on-going. This capability is intentional to adapt to the highly likely aspect of change in a low cost university-led mission. As more subsystems reach maturity, the test campaign will be expanded for automated functional and thermal testing and more ATE will be hosted at the instrumentation bus. New test suites will use existing driver interfaces and thermo-functional test logic. 
TABLE IV: STATISTICAL SUMMARY OF CHANNEL SPECTRUM SPREAD

\begin{tabular}{|c|c|c|c|c|}
\hline Channel Metric & Max & Min & Mean & Std. Dev. \\
\hline \multicolumn{5}{|c|}{$24 \mathrm{dBm}$} \\
\hline Upper ACP (dBc) & -23.6 & -24.4 & -24.01 & 0.3116 \\
\hline Lower ACP (dBc) & -23.8 & -25.1 & -24.5 & 0.4487 \\
\hline OCBW (MHz) & 1.2512 & 1.2158 & 1.2290 & 0.0112 \\
\hline Freq. Error $(\mathrm{kHz})$ & 1.909 & -4.818 & -1.522 & 2.2590 \\
\hline \multicolumn{5}{|c|}{$26 \mathrm{dBm}$} \\
\hline Upper ACP (dBc) & -22.9 & -24.0 & -23.43 & 0.3875 \\
\hline Lower ACP (dBc) & -23.2 & -24.6 & -23.77 & 0.4836 \\
\hline OCBW (MHz) & 1.2604 & 1.2237 & 1.2419 & 0.0116 \\
\hline Freq. Error $(\mathrm{kHz})$ & 4.59 & -6.203 & -1.314 & 3.9512 \\
\hline \multicolumn{5}{|c|}{$28 \mathrm{dBm}$} \\
\hline Upper ACP (dBc) & -22.8 & -22.1 & -22.31 & 0.2390 \\
\hline Lower ACP $(\mathrm{dBc})$ & -22.2 & -23.0 & -22.44 & 0.2578 \\
\hline OCBW (MHz) & 1.2865 & 1.2502 & 1.2658 & 0.0107 \\
\hline Freq. Error $(\mathrm{kHz})$ & 0.591 & -7.454 & -2.261 & 2.7396 \\
\hline \multicolumn{5}{|c|}{$30 \mathrm{dBm}$} \\
\hline Upper ACP (dBc) & -21.1 & -24.0 & -22.45 & 1.0000 \\
\hline Lower ACP (dBc) & -21.1 & -24.1 & -22.49 & 1.0369 \\
\hline OCBW $(\mathrm{MHz})$ & 1.2978 & 1.2196 & 1.2584 & 0.0263 \\
\hline Freq. Error $(\mathrm{kHz})$ & 2.17 & -5.235 & -1.221 & 2.5089 \\
\hline
\end{tabular}

\section{ACKNOWLEDGMENT}

This project is funded by the National Research Foundation.

\section{REFERENCES}

[1] M. Swartwout, "The first one hundred cubesats: a statistical look," Journal of Small Satellites, vol. 2, no. 2, pp. 213-233, 2013.

[2] Operation Phakisa, http://www.operationphakisa.gov.za/operations/oel/ pmpg/pages/default.aspx

[3] J. Wagenknecht, S. Fredrickson, T. Manning, and B. Jones "Design, development and testing of the miniature autonomous extravehicular robotic camera (Mini AERCam) guidance, navigation, and control system," in Proc. of 26th Annual American Astronautical Society Guidance and Control Conference, AAS 2004, Breckenridge, CO, USA, Feb. 5-9, 2003.

[4] R. S. Kalawsky, J. O'Brien, S. Chong, C. Wong, H. Jia, H. Pan, and P. R. Moore, "Bridging the gaps in a model-based system engineering workflow by encompassing hardware-in-the-loop simulation," IEEE Systems J., vol. 7, no. 4, pp. 593-605, 2013.

[5] C. Paris, M. Parisse, and W. A. Allawi, "Thermovacuum tests on TIGRIsat structure," in Proc. of 2015 IEEE Metrology for Aerospace (MetroAeroSpace), Benevento, Italy, 160-165, June 4-5, 2015.

[6] S. Saponara, G. Casarosa, P. Hambloch, F. Ciuchi, L. Fanucci, and B. Sarti, Modeling, "Sensitivity analysis, and prototyping of low- $g$ acceleration acquisition systems for spacecraft testing and environmentalnoise measurements," IEEE Trans. on Instr. and Meas., vol. 60, no. 2, pp. 385-397, 2011.

[7] S. Plamauer and M. Langer, "Evaluation of micropython as application layer programming language on cubesats," in Proc. of 30th International Conference on Architecture of Computing Systems, ARCS 2017, Vienna, Austria, April 3-6, 2017.

[8] S. Kim, R. Elkis, and M. Peckerar, "Device verification testing of highspeed analog-to-digital converters in satellite communication systems," IEEE Trans. on Instr. and Meas., vol. 58, no. 2, pp. 270-280, 2009.

[9] L. Angrisani, M. D'Apuzzo, and M. Vadursi, "Power measurement in digital wireless communication systems through parametric spectral estimation," IEEE Trans. on Instr. and Meas., vol. 55, no. 4, pp. 1051$1058,2006$.

[10] K. M. Gharaibeh, K. G. Gard, and M. B. Steer, "Accurate estimation of digital communication system metrics - SNR, EVM and $\rho$ in a nonlinear amplifier environment," in Proc. of Microwave Measurements Conference, Fall 2004. 64th ARFTG, pp. 41-44. Dec., 2004.
[11] V. Aparin, "Analysis of CDMA signal spectral regrowth and waveform quality," IEEE Trans. on Microwave Theory and Techniques, vol. 49, no. 12, pp. 2306-2314, 2001.

[12] A. Georgiadis, "Gain, phase imbalance, and phase noise effects on error vector magnitude," IEEE Trans. on Vehicular Tech., vol. 53, no. 2, pp. 443-449, 2004.

[13] J. M. Pieper, SCPI and VISA a valuable combination, ACEA, Wierden, The Netherlands. 


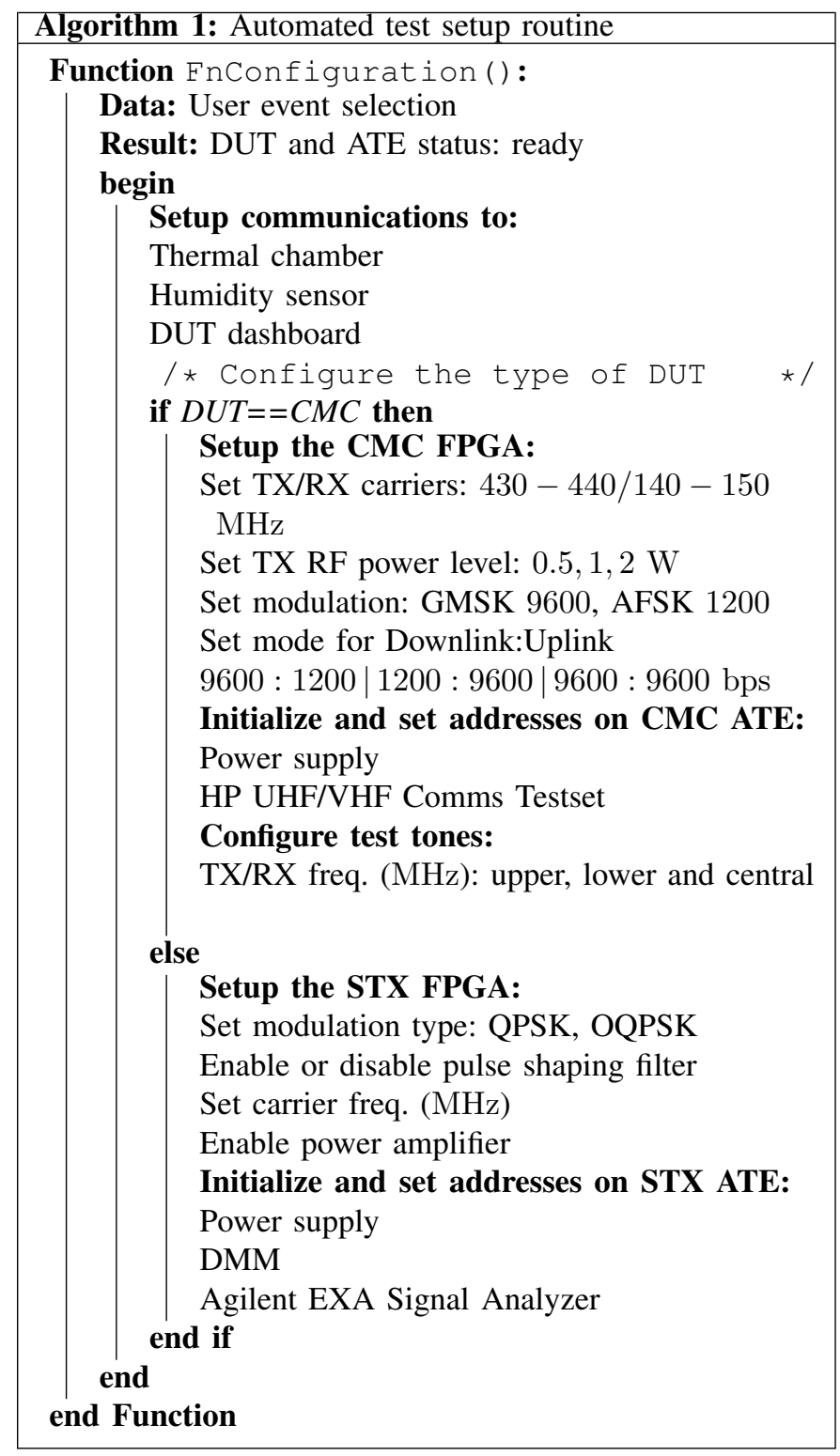

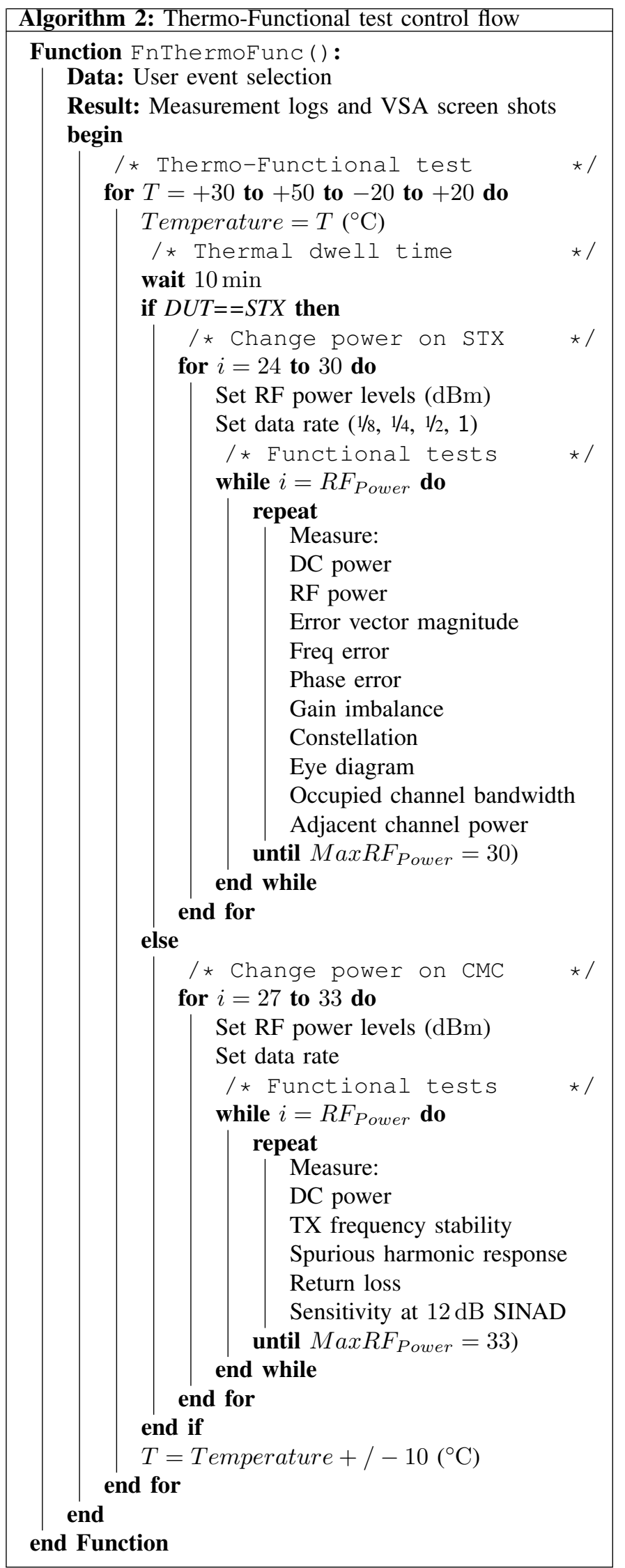

